\title{
Thermostatted Kinetic Models: Open Problems
}

\section{Carlo Bianca*}

\section{Dipartimento di Scienze Matematiche, Politecnico, Corso Duca degli Abruzzi 24, 10129 Torino, Italy}

This editorial article is concerned with some open problems regarding the thermostatted kinetic theory for active particles. This framework refers to the modeling of complex systems composed by a large number of elements grouped into different functional subsystems.

\section{Introduction}

The thermostatted kinetic theory for active particles has been recently proposed in [1] and generalized in papers [2-5], with the aim of modeling real-world complex systems. Complexity is an intrinsic characteristic of most living systems that makes the modeling of the system complicated. Indeed not only the large number of elements constituting the system but also the emergence of behaviour that arises as result of the whole interactions among the elements that occur in nonlinear fashion are key issues of the complexity. The complex behavior is also due to the fact that the living entities, differently from the inert matter entities, have the ability to perform specific strategies and functions such that small variations in their will could modify the overall asymptotic dynamics. According to the thermostatted theory for active particles, the complex system is composed by a large number of elements, called active particles that interact in nonlinear matter and under the effect of macroscopic external force fields. The microscopic state of the active particles is composed, at time $t$, by the space variable $\mathrm{x}$, the velocity variable $\mathrm{v}$, and the activity variable $\mathrm{u} \in \mathrm{D}_{\mathrm{u}}$ which models the ability of the particles to express strategy. Particles having the same strategy are grouped into a subsystem, called functional subsystem. The evolution of each functional subsystem is depicted by a distribution function $\mathrm{f}_{\mathrm{i}}(\mathrm{t} ; \mathrm{x} ; \mathrm{v} ; \mathrm{u})$ defined on $[0 ; \mathrm{T}] \times \mathrm{D}_{\mathrm{x}} \times \mathrm{D}_{\mathrm{v}} \times \mathrm{D}_{\mathrm{u}}$. In particular $\mathrm{f}_{\mathrm{i}}(\mathrm{t}$; $\mathrm{x} ; \mathrm{v} ; \mathrm{u}) \mathrm{dx} d \mathrm{v}$ du stands for the density of particles into the volume element $d x d v d u$ centered at $(x ; v ; u)$. It is worth stressing that every function defined in this editorial article is assumed to be measurable in all variables.

\section{The mathematical framework}

Let $\Omega=\mathrm{D}_{\mathrm{x}} \times \mathrm{D}_{\mathrm{v}} \times \mathrm{D}_{\mathrm{u}}$ be the domain of the all possible microscopic states of particles. Each functional subsystem is subjected to the external force field $\mathrm{F}_{\mathrm{i}}=\mathrm{F}_{\mathrm{i}}(\mathrm{u}): \mathrm{Du} \rightarrow \mathrm{R}$ whose magnitude modifies the asymptotic behavior and move out of equilibrium the system. The function $F_{i}$ is assumed to be a known function of $u$. The evolution equation for the distribution function $\mathrm{f}_{\mathrm{i}}$ is obtained under the following main assumptions:

- The interactions among the particles occur with rate $n_{i j}$, which denote the probability that a particle of the ith functional subsystem with activity $\mathrm{u}_{*}$ interacts with a particle of the jth functional subsystem with activity $\mathrm{u}^{*}$.

- The particles of the ith functional subsystem with activity $\mathrm{u}_{*}$ interacting with the particles of the $\mathrm{jth}$ functional subsystem with activity $\mathrm{u}^{*}$ have a probability density to reach the activity $\mathrm{u}$ given by $\mathrm{A}=\mathrm{A}\left(\mathrm{u}_{*} ; \mathrm{u}^{*} ; \mathrm{u}\right)$, which satisfies the following condition:

$$
\int_{D_{u}} A\left(u_{*}, u^{*}, u\right) d u=1, \forall u_{*}, u^{*} \in D_{u}
$$

- A particle moves with constant velocity $\mathrm{v} \in \mathrm{D}_{\mathrm{v}}$ into a straight line, stops after a certain time, chooses a new direction, continues running and so on (velocity-jump process). The domain $\mathrm{D}_{\mathrm{v}}$ is such that $\mathrm{v}$ and $-\mathrm{v}$ $\left.\in D_{v}\right)$. The thermostatted kinetic theory for active particles for $f_{i}, i \in\{1$, $2, \ldots, \mathrm{n}\}$, thus reads:

$$
\left(\partial_{t}+v \cdot \nabla_{x}\right) f_{i}+\partial u\left(F_{i}(u)\left(1-u \int_{\Omega} u \tilde{f} d x d v d u\right) f_{i}\right)=J_{i}[f]+v V_{i}\left[f_{i}\right]
$$

where

$$
\tilde{f}(t, x, v, u)=\sum_{i=1}^{n} f_{i}(t, x, v, u)
$$

and

$\cdot \mathrm{v} \cdot \nabla_{\mathrm{x}} \mathrm{fi}$ is the usual transport operator;

- $v$ is the turning rate or turning frequency of the velocity-jump, hence $1 / v$ is the mean run time;

- The operator $J_{i}[f]=J_{i}[f](t ; x ; v ; u)$ models the interactions in the activity, and reads:

$$
\begin{aligned}
& J_{i}[f]=\sum_{j=1}^{n} \int_{D_{u} \times D_{u}} \eta_{i j} A_{i j}\left(u_{*}, u^{*}, u\right) f_{i}\left(t, x, v, u_{*}\right) f_{j}\left(t, x, v, u^{*}\right) d u_{*} d u^{*}- \\
& f_{i}(t, x, v, u) \sum_{j=1}^{n} \int_{D u} \eta_{i j} f_{j}\left(t, x, v, u^{*}\right) d u^{*}
\end{aligned}
$$

The above term is obtained by considering the following rule: a particle with state $\mathrm{u}$ having an encounter with a particle with state $\mathrm{u}^{\star}$ will change its activity as a result of the interaction and thus will leave the state $\mathrm{u}$. The operator $\mathrm{V}_{\mathrm{i}}\left[\mathrm{f}_{\mathrm{i}}\right] \equiv \mathrm{V}_{\mathrm{i}}\left[\mathrm{f}_{\mathrm{i}}\right](\mathrm{t} ; \mathrm{x} ; \mathrm{v} ; \mathrm{u})$, which models the velocity-jump process is defined as follows:

$$
V_{i}\left[f_{i}\right]=\int_{D_{v}}\left[T_{i}\left(v^{*}, v\right) f_{i}\left(t, x, v^{*}, u\right)-T i\left(v, v^{*}\right) f_{i}(t, x, v, u)\right] d v^{*}
$$

where $\mathrm{T}_{\mathrm{i}}\left(\mathrm{v}^{*}, \mathrm{v}\right)$ is the turning kernel which give the probability that the velocity $\mathrm{v}^{\star} \in \mathrm{D}_{\mathrm{v}}$ jumps into the velocity $\mathrm{v} \in \mathrm{D}_{\mathrm{v}}$ (if a jump occurs). The transport term due to the activity of the particles reads:

$$
T F_{i}\left[f_{i}\right]:=\partial u\left(F i(u)\left(1-u \int_{\Omega} u \tilde{f}(t, x, v, u) d x d v d u\right) f_{i}(t, x, v, u)\right)
$$

This term models the Gaussian thermostat, which is based on the mathematical thermostats [6,7] and the Gauss's principle of the least constraint [8].

\section{Open Challenges}

The thermostatted kinetic framework proposed in this editorial article generalizes the thermostatted kinetic frameworks of the

*Corresponding author: Carlo Bianca, Dipartimento di Scienze Matematiche Politecnico, Corso Duca degli Abruzzi 24, 10129 Torino, Italy, E-mail: 016317@polito.it

Received December 03, 2013; Accepted December 04, 2013; Published December 10, 2013

Citation: Bianca C (2013) Thermostatted Kinetic Models: Open Problems. J App Computat Math 2: e133. doi:10.4172/2168-9679.1000e133

Copyright: (c) 2013 Bianca C. This is an open-access article distributed under the terms of the Creative Commons Attribution License, which permits unrestricted use, distribution, and reproduction in any medium, provided the original author and source are credited. 
pertinent literature; see the recent review paper [9], to complex systems where the space and velocity variables have an important role. Nevertheless we are aware that mathematical problems are still open. Specifically: - The first open problem is the existence and uniqueness of solution of the main framework (1.2). This is a hard problem considering that the presence of the transport term, namely the role of the space and velocity variables, and the nonlinearity introduced by the thermostat term. The second open problem refers to the modeling of biological systems, where genetic mutations can modify the interactions among the cancer cells, therefore the underlying description offered by the thermostatted kinetic theory models needs to be related to the evolution at the molecular (microscopic) and tissue (macroscopic) scale. In this context the asymptotic limits methods can be performed, see papers [10-12] and references cited therein. - The third open problem refers to the environment role. Indeed, as known, the collective behavior of biological, animal or human systems occurs in response to environmental factors that can affect the whole dynamics. Therefore the environment role has to be taken into account, not only at the macroscopic scale by $\mathrm{F}$, but also by modeling the interaction with the outer environment at the microscopic scale. Modeling external actions at the microscopic scale means representing the outer system as a specific functional subsystem with the ability to interact with the active particles of the inner system. It is worth stressing that the OMICS' Applied \& Computational Mathematics Open Access policy (http://www. omicsonline.org/OpenAccess.php) and the special features provided by the publisher (http://www.omicsonline.org/ specialfeatures.php) can help the community to give an answer to these open challenge.

\section{References}

1. Bianca C (2012) Kinetic theory for active particles modeling coupled to Gaussian thermostats. Applied Mathematical Sciences 6: 651-660.

2. Bianca C (2013) Controllability in hybrid kinetic equations modeling nonequilibrium multicellular systems. The Scientific World Journal 274719

3. Bianca C, Ferrara M, Guerrini L (2013) High-order moments conservation in thermostatted kinetic models. Journal of Global Optimization.

4. Bianca C (2013) Modeling complex systems with particles refuge by Thermostatted kinetic theory methods. Abstract and Applied Analysis 152174.

5. Bianca C, Dogbe C, Guerrini L (2013) A thermostatted kinetic framework with particle refuge for the modeling of tumor hiding. Applied Mathematics \& Information Sciences 8: 469-473.

6. Morris GP, Dettmann CP (1998) Thermostats: analysis and application. Chaos 8: 321-336.

7. Ruelle D (1998) Smooth dynamics and new theoretical ideas in non equilibrium statistical mechanics. J Stat Phys 95: 393-468.

8. GaussKF(1829)UbereinNeuesAllgemeinesGrundgesatz der Mechanik (On a New Fundamental Law of Mechanics). J ReineAngewandte Mat 4: 232-235.

9. Bianca C (2012) Thermostatted kinetic equations as models for complex systems in physics and life sciences. Physics of Life Reviews 9: 359-399.

10. BellouquidA, Bianca C (2010) Modelling aggregation-fragmentation phenomena from kinetic to macroscopic scales. Math Comput Modelling 52: 802-813.

11. Dogbe C (2008) Fluid dynamic limits for gas mixture I: Formal derivations. Math Models Methods Appl Sci 18: 1633-1672.

12. DegondP, Wennberg B (2007) Mass and energy balance laws derived from high-field limits of thermostatted Boltzmann equations. Commun Math Sci 5 : 355-382. 